\title{
Effect of Vallecular Ballooning in Stroke Patients With Dysphagia
}

\author{
Yong Kyun Kim, MD, PhD, Sang-heon Lee, MD, Jang-won Lee, MD
}

Department of Physical Medicine and Rehabilitation, Myongji Hospital, Goyang, Korea

\begin{abstract}
Objective To investigate the improvement of dysphagia after balloon dilatation and balloon swallowing at the vallecular space with a Foley catheter in stroke patients.

Methods This study was conducted between May 1, 2012 and December 31, 2015, and involved 30 stroke patients with complaints of difficulty in swallowing. All patients underwent videofluoroscopic swallowing study (VFSS) before and after vallecular ballooning. VFSS was performed with a $4 \mathrm{~mL}$ semisolid bolus. For vallecular ballooning, two trainings were performed for at least 10 minutes, including backward stretching of the epiglottis and swallowing of a balloon located in the vallecular space, by checking the movement of the Foley catheter tip in real time using VFSS.

Results After examination of the dysphagia improvement pattern before and after vallecular ballooning, laryngeal elevation ( $\mathrm{x}$-axis: pre $2.62 \pm 1.51 \mathrm{~mm}$ and post $3.54 \pm 1.93 \mathrm{~mm}, \mathrm{p}=0.038$; $\mathrm{y}$-axis: pre $17.11 \pm 4.24 \mathrm{~mm}$ and post $22.11 \pm 3.46 \mathrm{~mm}, \mathrm{p}=0.036$ ), pharyngeal transit time (pre $5.76 \pm 6.61 \mathrm{~s}$ and post $4.08 \pm 5.49 \mathrm{~s}, \mathrm{p}=0.043$ ), rotation of the epiglottis (pre $53.24^{\circ} \pm 26.77^{\circ}$ and post $32.45^{\circ} \pm 24.60^{\circ}, \mathrm{p}<0.001$ ), and post-swallow pharyngeal remnant (pre $41.31 \% \pm 23.77 \%$ and post $32.45 \% \pm 24.60 \%, p=0.002$ ) showed statistically significant differences. No significant difference was observed in the penetration-aspiration scale score (pre $4.73 \pm 1.50$ and post $4.46 \pm 1.78, \mathrm{p}=0.391$ ). Conclusion For stroke patients with dysmotility of the epiglottis and post-swallowing residue, vallecular ballooning can be considered as an alternative method that can be applied without risk of aspiration in dysphagia treatment.
\end{abstract}

Keywords Stroke, Deglutition disorder, Fluoroscopy, Epiglottis

Received May 11, 2016; Accepted August 17, 2016

Corresponding author: Jang-won Lee

Department of Physical Medicine and Rehabilitation, Myongji Hospital, 55 Hwasu-ro 14beon-gil, Deokyang-gu, Goyang 10475, Korea. Tel: +82-31-8106450, Fax: +82-31-810-5259, E-mail: rallo1080@naver.com

ORCID: Yong Kyun Kim (http://orcid.org/0000-0002-1224-8965); Sang-heon Lee (http://orcid.org/0000-0003-2770-8562); Jang-won Lee (http://orcid. org/0000-0002-2091-6150).

(c) This is an open-access article distributed under the terms of the Creative Commons Attribution Non-Commercial License (http://creativecommons.org/ licenses/by-nc/4.0) which permits unrestricted noncommercial use, distribution, and reproduction in any medium, provided the original work is properly cited. Copyright ( 2017 by Korean Academy of Rehabilitation Medicine 


\section{INTRODUCTION}

Swallowing involves the passive movement of the epiglottis by the force of the muscles attached to it, pulling the tip posteriorly so that it assumes a horizontal tilt and bends the tip downward [1].

The tip and sides of the epiglottis are primarily attached to the hyoid bone, while the base of the epiglottis is attached to the thyroid cartilage ligament. As the hyoid and thyroid cartilage are elevated during swallowing, the epiglottis moves to a horizontal and then inverted position, covering the arytenoids and vocal folds. The base of the tongue then presses against the lingual surface of the epiglottis, helping to squeeze out the contents of the valleculae [2].

Any deterioration of the epiglottis movement leads to the development of dysphagia. According to Garon et al. [3], the deterioration of epiglottic movement appears in stroke and Parkinson disease, chronic obstructive pulmonary disease (COPD), Alzheimer disease and muscular dystrophy, etc. Many epiglottic movement patterns have been described in the literature by earlier swallow researchers. The descriptions of abnormal epiglottic physiology includes no functional movement, incomplete inversion to a horizontal position, delayed tilt, oblique movement, and inconsistent neural innervationepiglottic dysfunction during deglutition in patents with dysphagia [4].

Perlman et al. [5] mentioned that in their study that deterioration in the movement of the epiglottis was associated with the vallecular residue and increased aspiration risk. They also reported that epiglottis dysfunction is associated with reduced hyoid elevation. Several techniques have been suggested to alleviate the deteriorated movement of the epiglottis and hyoid bone movement, including the Shaker exercise and Mendelsohn maneuver. Recently, an attempt was made to induce hyoid bone elevation by attaching Kinesio Tape to the chin and neck [6]. These methods improve the mobility of the epiglottis by promoting the laryngeal elevation.

A study by Kim et al. [7] reports the improvement of the swallowing parameters, including reduction of the vallecular remnant and decrease in pharyngeal transit time, after backward stretching of the epiglottis through a ballooning procedure using a Foley catheter at the vallecular space in a patient with deteriorated kinetic movement of the epiglottis after intubation. The unique feature of this paper was that a swallowing improvement effect was obtained by imposing direct passive backward stretching using ballooning to the epiglottis, unlike other previous papers that focused on improving the hyoid bone movement.

This study was conducted in stroke patients with dysphagia, and investigated the improvement of the epiglottis rotation angle, laryngeal elevation, post-swallowing remnant, and pharyngeal transit time, which were caused by the increase in the motility of the epiglottis after the ballooning procedure, using a Foley catheter at the vallecular space.

\section{MATERIALS AND METHODS}

The study was approved by the Myongji Hospital Ethics Committee. The Institutional Review Board number is MJH-16-033.

\section{Subjects}

This study involved 30 stroke patients with complaints of difficulty in swallowing. They were admitted to the rehabilitation medicine department, and underwent vallecular ballooning between May 1, 2012 and December 31,2015 . The inclusion criteria were neurogenic dysphagia patients with cerebral hemorrhage and cerebral infarction with vallecular ballooning procedure. The exclusion criteria were as follows: (1) patients with anatomical abnormality in the epiglottis, as observed on laryngoscopy conducted before the procedure; (2) patients with a treatment history that might affect the epiglottis mobility, such as head and neck radiation therapy; and (3) patients with a history of epiglottitis.

\section{Procedure}

Videofluoroscopic swallowing study (VFSS) was performed before and after vallecular ballooning, with a 4 $\mathrm{mL}$ semisolid bolus. The semisolid paste was prepared as a 2:1 volumetric mixture of dysphagia formula level 1 (food should be pudding-like without lumps, chunks, or pulp) and contrast barium solution (Solotop-HD; Taejoon Pharm Co. Ltd., Yongin, Korea). For the contrast solution, $375 \mathrm{~g}$ barium sulfate powder was mixed with $90 \mathrm{~mL}$ water. The VFSS test was done in the chin-tucked position for all the patients while they were sitting in a 
reclining wheelchair.

For the vallecular balloon procedure, contrast medium was injected to the Foley after placement of a 16 F urethral catheter tip in the epiglottis vallecular space. Stretching and spreading of the epiglottis 10 times for 10 seconds per time, was done by ballooning up to $6 \mathrm{~mL}$, while checking the balloon size with a VFSS image. Next, the patients had a 10-second break, and the same procedure was repeated three times. Additionally, the patients swallowed the balloon located at the vallecular space more than 20 times, which lasted for at least 10 minutes (Fig. 1). For the patients who were unable to follow the instructions due to cognitive decline, the swallowing reflex was induced by manual stimulation with the index and middle fingers after palpating the upper hyoid bone, after which the balloon swallowing was conducted.

Every VFSS was recorded using a camcorder at a rate of 30 frames per second. All images were saved on a personal computer, and were analyzed with a multimedia player (Gretech, Seoul, Korea). Additionally, the laryn- geal elevation, post-swallow pharyngeal remnant, and epiglottis rotation angle were measured with 2D screen AutoCAD (Autodesk, San Francisco, CA, USA). As the forward, backward, left and right movements of the patient during the VFSS test might affect the measurements of the remnant and width, to minimize the error the image size was readjusted based on the maximum length of a 100 -won coin ( $23 \mathrm{~mm}$ in diameter) attached to the neck of a patient.

\section{Laryngoscopic evaluation of the epiglottis}

To identify any complications at the site where vallecular ballooning was imposed (including mucosal injury), evaluation was conducted with a fiberoptic rhinolaryngoscope (ENF-GP; Olympus Medical Systems, Tokyo, Japan) after the procedure.

\section{Outcome measures}

The swallowing parameters were measured as described in a previous study by Kim et al. [8]. For laryngeal
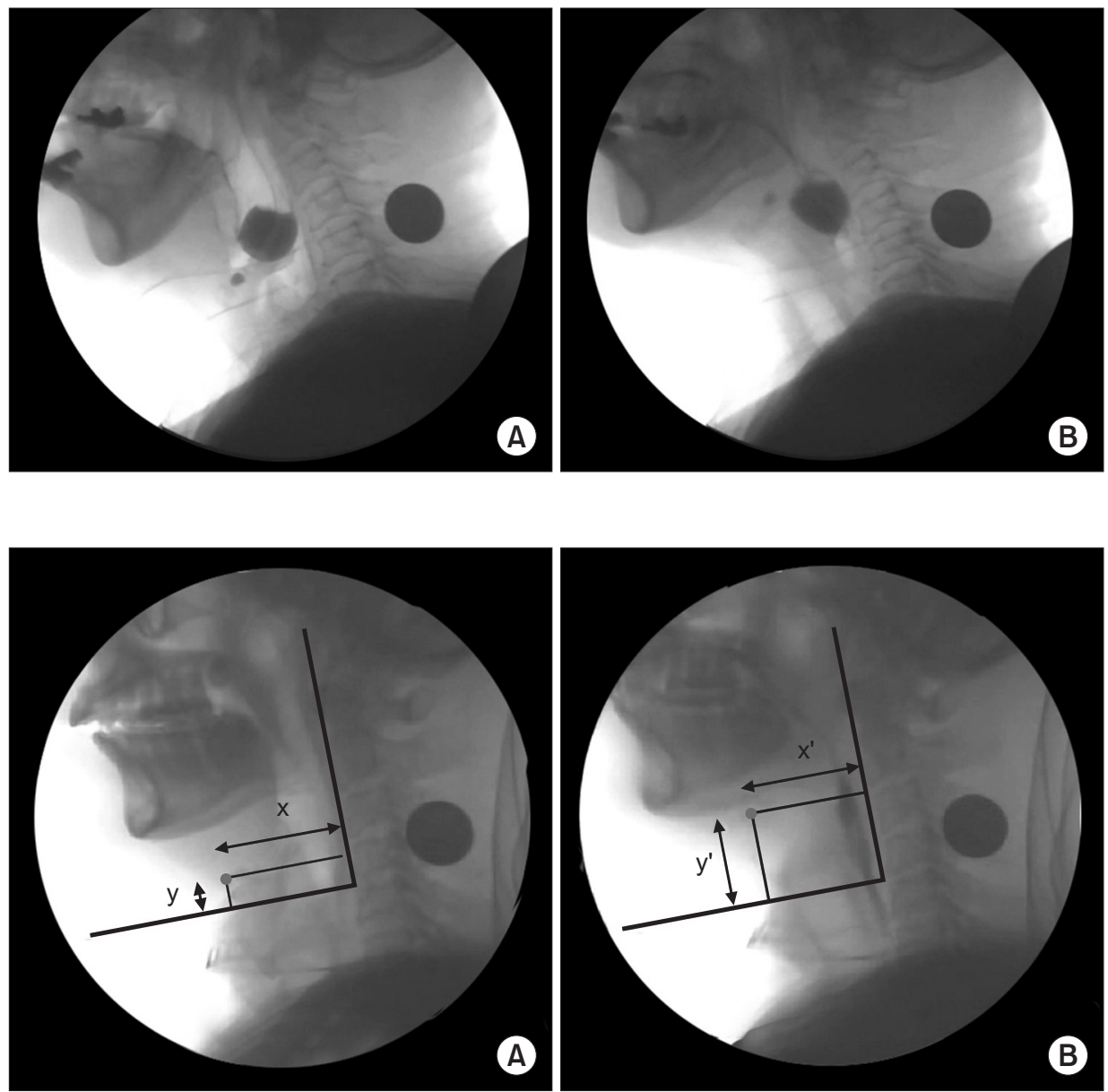

Fig. 1. Vallecular ballooning. Contrast medium was injected into the balloon after inserting the tip into the vallecular space by checking the movement of the tip of the Foley catheter in real time using videofluoroscopic swallowing study. Two kinds of training were performed: backward stretching of the epiglottis (A) and swallowing of a balloon located in the vallecular space (B).

Fig. 2. Laryngeal elevation. The zero point was defined as the anteriorinferior margin of the 4 th cervical vertebral body, the y-axis as the straight line connecting the zero point with the anterior-inferior margin of the second cervical vertebral body, and the $\mathrm{x}$-axis as the line perpendicular to the y-axis. Hyoid bone at the position of the resting state (A) and most highly raised position during swallowing (B). 
elevation, the $y$-axis was a straight line drawn from the anterior-inferior margin of the 4 th vertebral body to the anterior-inferior margin of the 2 nd vertebral body. The $\mathrm{x}$-axis was set perpendicular to the $\mathrm{y}$-axis to measure the change $(\mathrm{mm})$ in the location of the hyoid bone before and after swallowing (Fig. 2). The pharyngeal transit time was measured as the time (second) that it took for food to pass from the posterior nasal spine to the pharyngoesophageal sphincter. The post-swallow pharyngeal remnant was represented as the difference between the initial remnant and the remnant after swallowing the food once (Fig. 3). The degree of semisolid aspiration was measured using the penetration-aspiration scale (PAS). After the epiglottis rotation angle was checked with an imaginary line connecting the root and tip of the epiglottis, the angle change in the imaginary line before and after swallowing was measured. To reduce error, the angle change was measured based on a straight line connecting the anterior-inferior margin of the 4th vertebral body and the anterior-inferior margin of the 2 nd vertebral body (Fig. 4).

\section{Statistical analysis}

All statistical analyses were performed with the SPSS ver. 18.0 (SPSS Inc., Chicago, IL USA). Statistical significance was confirmed with the paired t-test for comparing the results before and after the balloon treatment. A pvalue $<0.05$ was considered to be statistically significant.

\section{RESULTS}

The subjects included 18 males $(60 \%)$ and 12 females (40\%) with mean age of $49.21 \pm 16.51$ years. The type of stroke was hemorrhage for 14 patients $(46.67 \%)$ and infarction for 16 patients (53.33\%). With regards to the involved side, the right hemisphere was affected in 14 patients (46.67\%), left in 7 patients (23.33\%), and others for
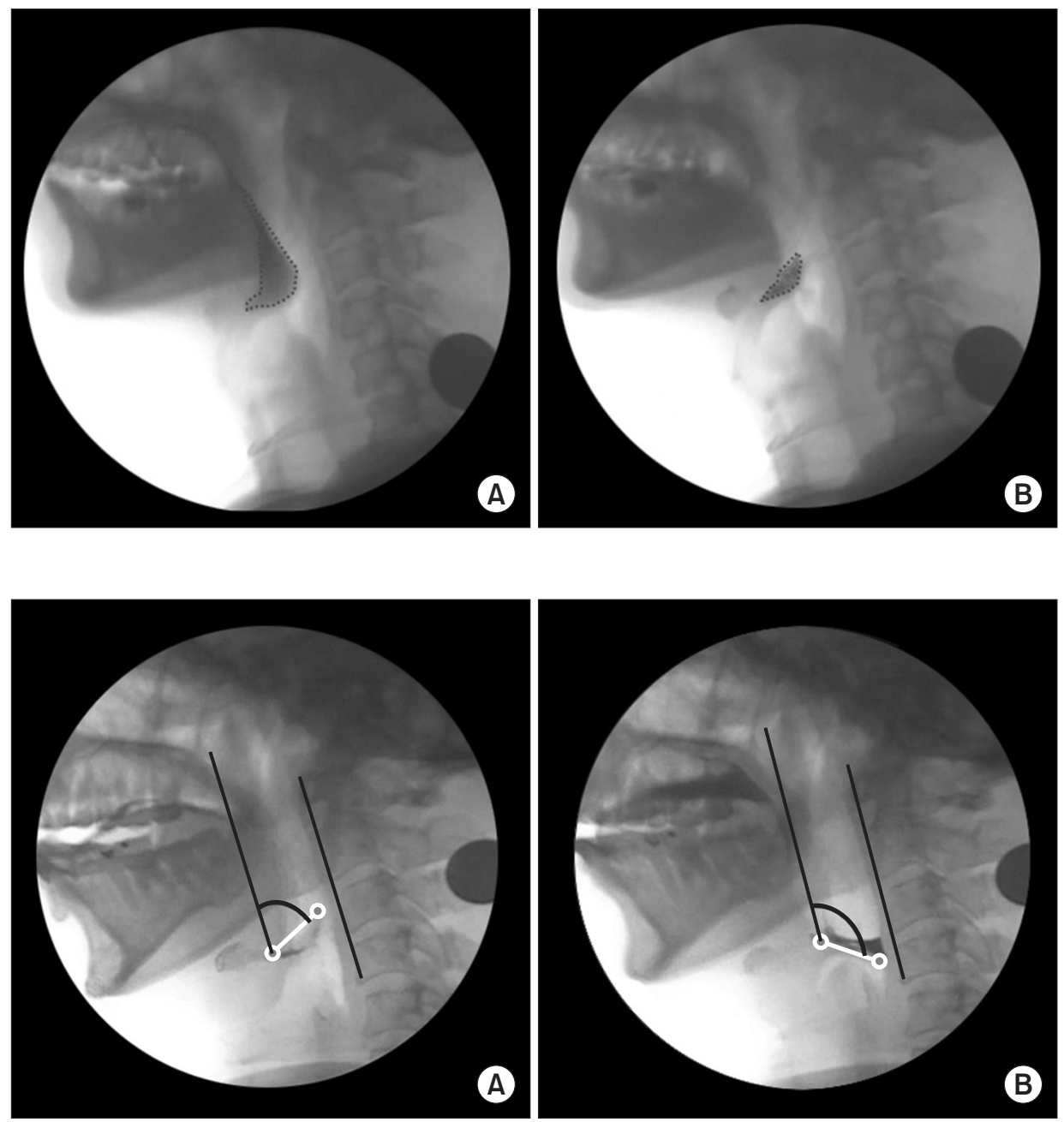

Fig. 3. Post-swallow pharyngeal remnant. The difference in the remnant (\%) was measured before and after swallowing, using an AutoCAD 2D screen. Remnant before swallowing (A) and remnant after swallowing (B).

Fig. 4. Epiglottis rotation. The maximum angle change in the line connecting the root with the tip of the epiglottis before and after swallowing was measured. To reduce error, a straight line linking the anteriorinferior margin of the 4 th vertebral body and the anterior-inferior margin of the 2 nd vertebral body was used as a baseline. Before swallowing (A) and maximum angle during swallowing (B). 
9 patients (30\%). Other areas include the brain stem, cerebellum, and multiple areas. The time from stroke onset to VFSS was $378.70 \pm 207.70$ days and the mean K-MMSE (Korean version of Mini-Mental Status Examination) score was $5.70 \pm 6.20$ points. The PAS score was $4.73 \pm 1.50$ points (Table 1).

Observation of improvement pattern of dysphagia after vallecular ballooning revealed laryngeal elevation ( $\mathrm{x}$-axis: pre $2.62 \pm 1.51 \mathrm{~mm}$ and post $3.54 \pm 1.93 \mathrm{~mm}, \mathrm{p}=0.038$; $\mathrm{y}$-axis: pre $17.11 \pm 4.24 \mathrm{~mm}$ and post $22.11 \pm 3.46 \mathrm{~mm}, \mathrm{p}=0.036$ ),

Table 1. Basic characteristics of the subjects

\begin{tabular}{lc}
\hline \multicolumn{1}{c}{ Characteristic } & Value \\
\hline Age (yr) & $49.21 \pm 16.51$ \\
Gender & $18(60)$ \\
Male & $12(40)$ \\
Female & \\
Type of stroke & $14(46.67)$ \\
Hemorrhagic & $16(53.33)$ \\
Ischemic & $14(46.67)$ \\
Affected hemisphere & $7(23.33)$ \\
Right & $9(30)$ \\
Left & $378.70 \pm 207.70$ \\
Others ${ }^{\text {a) }}$ & $5.70 \pm 6.20$ \\
Time from stroke onset to VFSS (day) & $4.73 \pm 1.50$ \\
\hline K-MMSE & \\
\hline
\end{tabular}

Values are presented as mean \pm standard deviations or number (\%).

K-MMSE, Korean Mini-Mental State Examination; PAS, penetration-aspiration scale.

${ }^{\text {a) }}$ Other areas include brain stem, cerebellum, and multiple areas. pharyngeal transit time (pre 5.76 \pm 6.61 seconds and post $4.08 \pm 5.49$ seconds, $\mathrm{p}=0.043$ ), rotation of the epiglottis (pre $53.24^{\circ} \pm 26.77^{\circ}$ and post $\left.32.45^{\circ} \pm 24.60^{\circ}, \mathrm{p}<0.001\right)$, and postswallow pharyngeal remnant (pre $41.31 \% \pm 23.77 \%$ and post $32.45 \% \pm 24.60 \%, p=0.002$ ) had statistically significant differences before and after the procedure. No statistical significance was observed for semisolid aspiration (PAS score: pre $4.73 \pm 1.50$ and post $4.46 \pm 1.78, \mathrm{p}=0.391$ ) (Table 2).

\section{DISCUSSION}

Foods are delivered safely and effectively to the stomach through the complex process of swallowing, which involves the elaborate adjustment of movements from the mouth to the esophagus within a short period of time, and requires well-coordinated and sequential contraction and relaxation of the mouth and pharyngeal muscles [9].

Logemann [10] divided the normal swallowing process into 4 steps: the oral preparation, followed by the oral, pharyngeal, and esophageal steps. In the pharyngeal step, the epiglottis backward stretching is achieved through the rise of the hyoid bone. Movement occurs when the epiglottis covers the larynx, and consequently, the airway is complexly closed.

Garon et al. [3] investigated the abnormal epiglottic movement pattern in patients with dysphagia, and reported that epiglottic dysmotility can be caused by several factors such as COPD, Parkinson disease, amyotrophic lateral sclerosis (ALS), and dementia, and including stroke as one of the factors. The abnormal epiglottic movement pattern is not a functional movement, incomplete inversion to a horizontal position, delayed tilt,

Table 2. The improvement pattern of dysphagia before and after vallecular ballooning

\begin{tabular}{lcccc}
\hline \multicolumn{1}{c}{ Variable } & Before ballooning & After ballooning & p-value \\
\hline Laryngeal elevation x-axis (mm) & $2.62 \pm 1.51$ & $3.54 \pm 1.93$ & $0.038^{*}$ \\
\hline Laryngeal elevation y-axis (mm) & $17.11 \pm 4.24$ & $22.11 \pm 3.46$ & $0.036^{*}$ \\
Pharyngeal transit time (s) & $5.76 \pm 6.61$ & $4.08 \pm 5.49$ & $0.043^{*}$ \\
Rotation of the epiglottis ( $\left.{ }^{\circ}\right)$ & $53.24 \pm 26.77$ & $72.51 \pm 40.34$ & $<0.001^{*}$ \\
\hline Post-swallow remnant (\%) & $41.31 \pm 23.77$ & $32.45 \pm 24.60$ & $0.002^{*}$ \\
Semisolid aspiration (PAS score) & $4.73 \pm 1.50$ & $4.46 \pm 1.78$ & 0.391 \\
\hline
\end{tabular}

Values are presented as mean \pm standard deviations.

PAS, Penetration-Aspiration Scale.

${ }^{*} \mathrm{p}<0.05$ by paired $\mathrm{t}$-test (before vs. after vallecular ballooning). 
oblique movement, mix pattern, etc.

Epiglottis is a cartilage not attached to the muscles, but attached to the hyoid bone, and is reported to be immobile by itself, moving passively through the hyoid bone movement, namely laryngeal elevation [11]. In addition, Perlman et al. [5] reported that epiglottis dysfunction, reduced hyoid elevation, and deteriorated oromotor function affects the vallecular residue. Also, the hyoid elevation was associated with epiglottis dysfunction.

Compensation techniques for laryngeal elevation include the Shaker exercise and Mendelsohn maneuver, effortful swallowing, and multiple swallowing. Efforts to solve abnormal epiglottis movement have been made with the help of above procedures [12].

Shaker exercise narrows the space between the tongue base and the posterior wall of the pharynx. It increases the pharyngeal pressure so that the bolus can easily pass to the pharynx [13]. Additionally, in patients with weakened laryngeal elevator muscles and late swallowing reflex, it reduces the risk of immediate aspiration of the bolus to the airway before swallowing, by inducing laryngeal elevation and epiglottis backward stretching [14]. The Mendelsohn maneuver maintains swallowing for 2-3 seconds, slowly relaxing when the pharynx is elevated at maximum. It helps the remnants in the piriformis space to move to the esophagus, by helping in clearing the remnant in the vallecular space through laryngeal elevation and opening of the cricopharyngeal muscle [12].

A recent study showed that the aspiration rate was lowered through the hyoid elevation, and reduced the pharyngeal residue by inducing an increase in the oromotor function and swallowing pressure through lingual exercise [15]. In addition, Heo and Kim [6] investigated the changes in the hyoid bone elevation and epiglottis angular variation before and after the attachment of Kinesio Tape to the chin and neck, and reported an improvement in the vertical movement of the hyoid bone and rotation angle of the epiglottis, before and after the taping procedure. These methods, however, only induced epiglottis movement and were not direct epiglottis exercise methods.

Our institution had previously reported a case of improvement in the swallowing parameters, including reduction of the vallecular remnant and decrease in pharyngeal transit time, after epiglottis backward stretching through the ballooning procedure with a Foley catheter in the vallecular space in patients with lowered kinetic movement after intubation [7]. The subject of the previous study was not a patient of neurogenic dysphagia, and did not have disorders in the swallowing parameters, except for deteriorated epiglottis mobility.

This study was the first to examine the improvement of dysphagia by performing a direct procedure, using ballooning in the vallecular space in stroke patients with neurogenic dysphagia. Two kinds of training were performed for vallecular ballooning: backward stretching of the epiglottis, and swallowing of a balloon located in the vallecular space. Contrast medium was injected into the balloon after putting the tip into the vallecular space, by checking the movement of the tip of the Foley catheter in real time using VFSS.

Two outcomes were expected after the above procedures. One was improved motility by stretching of the epiglottis, and the other was strengthening of the geniohyoid, mylohyoid, and thyrohyoid muscles, which experienced laryngeal elevation caused by the swallowing of the balloon located in the vallecular space.

An increase in epiglottis angular movement was expected to be induced due to the stretching, with a subsequent increase in the bolus transit into the pyriformis space and improvement of the protection of the vocal cord of the epiglottis. With strengthening of the geniohyoid, mylohyoid, and thyrohyoid muscles, the effort for swallowing the balloon located in the vallecular space improves the laryngeal elevation and increases the swallowing pressure through the isometric contraction exercise of these muscles. Hence, an overall effect of reduced post swallowing remnant and decreased pharyngeal transit time were expected. In this study, the laryngeal elevation (anterior and vertical movement), angular rotation of the epiglottis, pharyngeal transit time, and post-swallow remnant decreased after performing the ballooning procedure in the vallecular space. Also, the PAS score showed improvement, but was not statistically significant, the reason for this being the various causes of aspiration. Aspiration is observed not only in patients with epiglottic dysmotility or post-swallow remnants, but also in patients with vocal cord palsy protecting the airway during swallowing [16] or in patients in whom the upper esophageal sphincter is not sufficiently relaxed or contracted during swallowing, such as in patients with cricopharyngeal dysfunction [17]. Therefore, if the subject group for vallecular ballooning 
is limited to patients complaining of dysphagia caused solely by epiglottis dysmotility, the aspiration is expected to improve. Further studies are required in this area.

The side effects expected after vallecular ballooning include vomiting, mucosal injury, and pain. During the procedure, no patient experienced vomiting. After the procedure, 26 of the 30 patients were evaluated. On laryngoscopy, no lesions were observed, including mucosal bleeding and erosion, and no patient complained of discomfort in swallowing after ballooning. Yoon's previous study that performed piriformis ballooning using a Foley catheter had also not reported observations of any adverse side effects [18]. Our hospital has conducted the piriformis and vallecular ballooning procedures using a Foley catheter since 4 years without reports of any side effects. However, this procedure may be affected by the practitioner and by the material of the Foley catheter, and long-term studies for safety are required.

The advantages of vallecular ballooning are as follows. First, there is no risk of aspiration. As training is conducted not with actual food but with a balloon, the risk of aspiration pneumonia is small. Second, the procedure can be conducted without any influence from the cognitive function. If the patients cannot follow instructions due to lowered cognitive function, the procedure can be performed by inducing the swallowing reflex through manual stimulation with the index and middle fingers after palpating the upper hyoid bone. Third, it can be conducted with minimal cost, using only a Foley catheter and VFSS.

The limitations of this study are as follows. Although improvement of the laryngeal elevation was observed, but it would have been beneficial if the changes in the direct values on the geniohyoid, mylohyoid, and thyrohyoid muscles had been examined using an electromyogram. The implementation of post-treatment VFSS right after balloon training is the easiest way to investigate the effect of balloon treatment. This study had the advantage of excluding the confounding factors, but it had limitations in investigating the changes that can be shown after long-term training. Since the evaluation is performed immediately after the intervention, there is a limitation that there is no study on the training effect. This study reflected the treatment performed in the first VFSS after admission, and investigated the short-term effects of balloon treatment. However, if the training is repeated for longer period of time, significant changes may be found in the other parameters. In future, a study to confirm the long-term effects needs to be designed.

In stroke patients with dysphagia, improvements in the laryngeal elevation (anterior and vertical movement), angular rotation of the epiglottis, pharyngeal transit time, and post-swallow remnant were observed after vallecular ballooning. In particular, vallecular ballooning for patients in whom epiglottic dysmotility and post-swallow residue are observed, can be considered as an alternative method to be applied without an aspiration risk in swallowing rehabilitation treatment.

\section{CONFLICT OF INTEREST}

No potential conflict of interest relevant to this article was reported.

\section{REFERENCES}

1. Fink BR, Martin RW, Rohrmann CA. Biomechanics of the human epiglottis. Acta Otolaryngol 1979;87:554-9.

2. Ekberg O, Sigurjonsson SV. Movement of the epiglottis during deglutition: a cineradiographic study. Gastrointest Radiol 1982;7:101-7.

3. Garon BR, Huang Z, Hommeyer M, Eckmann D, Stern GA, Ormiston C. Epiglottic dysfunction: abnormal epiglottic movement patterns. Dysphagia 2002;17:5768.

4. Ekberg O. Epiglottic dysfunction during deglutition in patients with dysphagia. Arch Otolaryngol 1983;109:376-80.

5. Perlman AL, Grayhack JP, Booth BM. The relationship of vallecular residue to oral involvement, reduced hyoid elevation, and epiglottic function. J Speech Hear Res 1992;35:734-41.

6. Heo SY, Kim KM. Immediate effects of Kinesio Taping on the movement of the hyoid bone and epiglottis during swallowing by stroke patients with dysphagia. J Phys Ther Sci 2015;27:3355-7.

7. Kim YK, Kim MT, Kim SK. The effect of balloon dilation at the vallecular using videofluoroscopic swallowing study on patient who has a dysphagia. Ann Rehabil Med 2013;37:426-9.

8. Kim YK, Choi JH, Yoon JG, Lee JW, Cho SS. Improved dysphagia after decannulation of tracheostomy in pa- 
tients with brain injuries. Ann Rehabil Med 2015;39: 778-85.

9. Cook IJ, Dodds WJ, Dantas RO, Kern MK, Massey BT, Shaker R, et al. Timing of videofluoroscopic, manometric events, and bolus transit during the oral and pharyngeal phases of swallowing. Dysphagia 1989;4:815.

10. Logemann JA. Evaluation and treatment of swallowing disorders. 2nd ed. Austin: Pro-ED; 1998. p. 38-50.

11. Ertekin C, Aydogdu I. Neurophysiology of swallowing. Clin Neurophysiol 2003;114:2226-44.

12. Ding R, Larson CR, Logemann JA, Rademaker AW. Surface electromyographic and electroglottographic studies in normal subjects under two swallow conditions: normal and during the Mendelsohn manuever. Dysphagia 2002;17:1-12.

13. Welch MV, Logemann JA, Rademaker AW, Kahrilas PJ. Changes in pharyngeal dimensions effected by chin tuck. Arch Phys Med Rehabil 1993;74:178-81.

14. Baek SS, Park SB, Lee SG, Lee KM, Kim SH. The effect of neck posture in swallowing of stroke patients. J Korean Acad Rehabil Med 1997;21:8-12.

15. Robbins J, Kays SA, Gangnon RE, Hind JA, Hewitt $\mathrm{AL}, \mathrm{Gentry} \mathrm{LR}$, et al. The effects of lingual exercise in stroke patients with dysphagia. Arch Phys Med Rehabil 2007;88:150-8.

16. Heitmiller RF, Tseng E, Jones B. Prevalence of aspiration and laryngeal penetration in patients with unilateral vocal fold motion impairment. Dysphagia 2000; 15:184-7.

17. Alfonsi E, Merlo IM, Ponzio M, Montomoli C, Tassorelli C, Biancardi C, et al. An electrophysiological approach to the diagnosis of neurogenic dysphagia: implications for botulinum toxin treatment. J Neurol Neurosurg Psychiatry 2010;81:54-60.

18. Kim YK, Choi SS, Choi JH, Yoon JG. Effectiveness of rehabilitative balloon swallowing treatment on upper esophageal sphincter relaxation and pharyngeal motility for neurogenic dysphagia. Ann Rehabil Med 2015;39:524-34. 